\title{
Experimental Study on Aerodynamic Heating Induced by Dual Injections into Hypersonic Cross Flow
}

\author{
Masato Taguchi, ${ }^{1}$ Koichi Mori, ${ }^{1}$ and Yoshiaki Nakamura ${ }^{2}$ \\ ${ }^{1}$ Department of Aerospace Engineering, Nagoya University, Nagoya 464-8601, Japan \\ ${ }^{2}$ Department of Mechanical Engineering, Chubu University, Kasugai 487-0027, Japan \\ Correspondence should be addressed to Masato Taguchi; taguchi@fluid.nuae.nagoya-u.ac.jp
}

Received 20 November 2016; Revised 12 March 2017; Accepted 28 March 2017; Published 8 May 2017

Academic Editor: William W. Liou

Copyright (c) 2017 Masato Taguchi et al. This is an open access article distributed under the Creative Commons Attribution License, which permits unrestricted use, distribution, and reproduction in any medium, provided the original work is properly cited.

\begin{abstract}
In this study, the distribution of surface heat transfer induced by dual side-jets injected into a hypersonic flow has been visualized using a temperature sensitive paint. The experiments were performed in both tandem and parallel injector arrangements, and the spacing between the injection holes was taken as a parameter in each arrangement. As a result, the aerodynamic heating in the separated region of the boundary layer and in the horseshoe vortex was clearly visualized. In the tandem arrangements, heat transfer remarkably increased immediately upstream of the front injector. The distributions and the intensity of surface heat transfer were similar to those caused by the single injection. On the other hand, in the parallel arrangements, the extent of the separation nearly doubled, and the maximum heat flux decreased to less than half of that from the single injection. The global distribution of heat transfer varied significantly as the injector spacing was changed. When the injectors were positioned with a large spacing, the interaction between the side-jets was relatively lowered, and thus distribution, as for the single injector, was induced around each injection hole individually. In contrast, with a short spacing, the dual injection behaved as a single obstacle. The most effective reduction of maximum heat flux was achieved with an intermediate injector spacing.
\end{abstract}

\section{Introduction}

Aerodynamic interaction induced by side-jet injection into supersonic and hypersonic cross flow has been investigated in a number of previous works [1]. The flow physics have an impact on applications such as reaction control systems (RCS) of rockets and fuel injection in scramjet engines.

Side-jet injection into hypersonic cross flow creates a complex, three-dimensional flow field; thus, the surface pressure and heat transfer are influenced by the interaction. A schematic of a typical flow field is shown in Figure 1. A barrel shock and Mach disc are formed in the jet plume. A bow shock is generated in the main flow upstream of the side-jet because the jet plume acts as an obstacle. The boundary layer upstream of the jet is separated due to an overpressure at the bow-shock site, and a separation shock is generated. Two different types of vortices are generated in the flow field: a counterrotating vortex (CRV) pair arising in the wake of the jet plume $[2,3]$ and a horseshoe vortex generated in the separation region in front of the sidejet, which develops downstream and wraps itself around the jet. The area of the separation region and number of horseshoe vortices are determined by conditions of uniformflow Reynolds number, the nozzle pressure ratio (NPR) of the jet, and the angle of attack of the model $[4,5]$. The horseshoe vortex in the vicinity of the wall induces surface heat transfer augmentation and reduction along its reattachment and separation lines, respectively $[4,6]$.

In several RCS concepts, multiple injections of the sidejets have been employed $[5,7,8]$. The flow field of multiple injections into hypersonic flow is much more complex than single injections because of the interaction between the jets [9]. Several studies have been conducted for fixedjet configurations in some specific designs. Watkins et al. visualized pressure and heat transfer distribution associated with a four-cluster yaw-jet RCS, which was mounted on the aft-body of an Orion concept configuration $[7,8]$. They reported that the four jets showed a relatively lower heat 


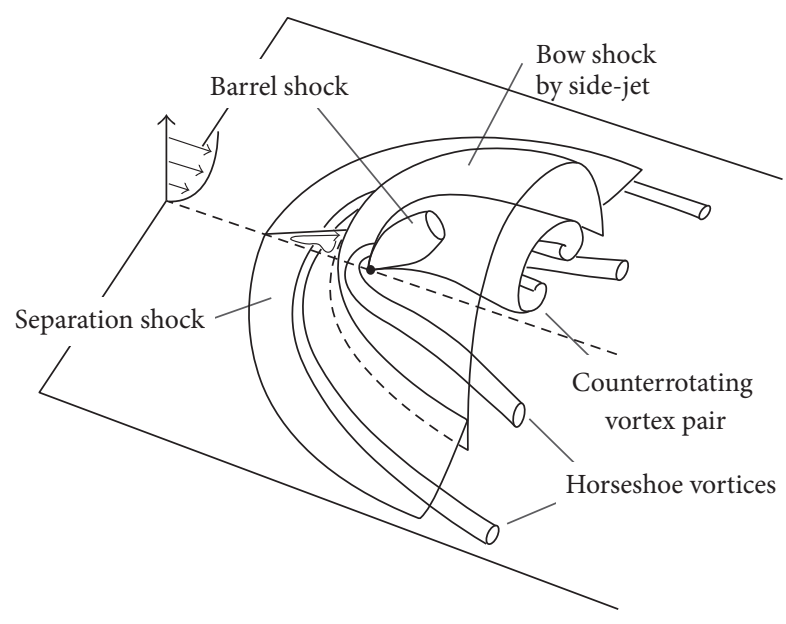

FIGURE 1: Schematic of a flow field associated with a single injection into hypersonic cross flow.

transfer increase, as compared to a conventional single jet, in terms of both the heating intensity and the extent. It was also reported that multiple injections in a "parallel configuration" (i.e., injection holes are aligned normal to the freestream) extended the separation area further upstream than single injections $[5,10]$. Pudsey et al. investigated four jets injected into hypersonic cross flow in a "tandem configuration" (i.e., injection holes are aligned streamwise). They suggested that a jet positioned behind another jet can restrict the expansion of the upstream jet, and this effect is relaxed as the jets spacing is increased [11].

The main purpose of this work is to understand the aerodynamic heating induced by dual injection. As mentioned above, several previous works have been performed that focused on multiple-injection interactions [5, 7-11]. From those results, it is expected that injector arrangements have a large influence on the associated interaction flow field and the surface pressure distribution. On the other hand, surface heat transfer has been investigated only for a single injector $[4,6]$, except for some application concepts $[7,8]$. The aerothermodynamic effect induced by multiple injections is thus still not understood systematically. This study focuses on changes of the aerodynamic heating intensity and its extent on the wall, caused by controlled dual-injector arrangements. Our main goal is to bridge the interaction of the flow field and the aerothermodynamic effect on the wall, which is associated with dual injection using several injector arrangements. It would thus be useful to estimate an optimum injector arrangement in terms of thermal load on the wall.

In the present study, two types of injector arrangements, tandem and parallel, on a hemisphere-cone model were investigated. This model was employed because interaction studies on this model with a single injection had been conducted under the same experimental condition in previous works $[12,13]$, both experimentally and numerically. Thus, the flow field on the model had been well understood for a single injection. The distance between the dual injectors was changed in three levels. Variations in surface heating patterns, configurations of shock systems, and heat transfer increase
TABLE 1: Test flow parameters.

\begin{tabular}{lcccc}
\hline $\begin{array}{l}\text { Mach } \\
\text { number, }\end{array}$ & $\begin{array}{c}\text { Total } \\
\text { pressure, } \\
M_{\infty}\end{array}$ & $\begin{array}{c}\text { Static } \\
\text { pressure, }\end{array}$ & $\begin{array}{c}\text { Total } \\
\text { temperature, }\end{array}$ & $\operatorname{Re}$ \\
\hline 8.1 & $4.0[\mathrm{MPa}]$ & $377[\mathrm{~Pa}]$ & $876[\mathrm{~K}]$ & $6.5 \times 10^{6}\left[\mathrm{~m}^{-1}\right]$ \\
\hline
\end{tabular}

(and reduction), based on the jets spacing, are described in detail. Surface heat transfer, which is represented in terms of Stanton number distribution and measured by means of temperature sensitive paint, is used to understand the interaction of the flow field. On the other hand, Schlieren visualization is used to visualize the shock system. The flow field associated with a single injector, under the test conditions in this study, is firstly discussed as a reference, before focusing on dual injectors.

\section{Experimental Setup}

2.1. Shock Tunnel. All tests were conducted in the reflected shock tunnel of Nagoya University. This wind tunnel consisted of a high-pressure driver tube $(6.5 \mathrm{~m})$, a driven tube $(10 \mathrm{~m})$, a Mach 8.1 hypersonic nozzle and a damp tank. The cross-sectional diameter of nozzle exit in the test section is $350 \mathrm{~mm}$. The dimensions are shown in Figure 2. The driver and driven tube are partitioned by two 1-mm thick aluminum diaphragms to create a medium-pressure section. Air is used as both driver and driven gas. Initial condition of the shock tunnel operation in each section was set as $4 \mathrm{MPa}$ in the highpressure driver tube, $2 \mathrm{MPa}$ in the medium-pressure section, $1 \mathrm{~atm}$ in the driven tube, and a vacuum $(\sim 6 \mathrm{~Pa})$ in the damp tank. When the shock tunnel starts, the medium pressure is released to atmosphere, and the two diaphragms rupture due to the pressure differential. A normal shock propagates downstream in the driven tube, and a gas of high pressure and temperature is created at the edge of the tube, which expands and accelerates through the hypersonic nozzle to Mach 8.1. The shock tunnel generates a test flow of Mach 8.1 for a duration of $30 \mathrm{~ms}$, with total pressure $P_{0}=4.0 \mathrm{MPa}$, total temperature $T_{0}=876 \mathrm{~K}$, and unit Reynolds number $\mathrm{Re}=6.5$ $\times 10^{6} \mathrm{~m}^{-1}$, as shown in Table 1 .

2.2. Wind Tunnel Model and Injection System. The dimensions of the wind tunnel model are shown in Figure 3 and schematic of the experimental system, in Figure 4. The test model had a hemisphere-cone configuration, with sphere radius $R=20 \mathrm{~mm}$, total body length $L=120 \mathrm{~mm}$, and half-angle cone $\theta=10^{\circ}$. It was fixed on the sting in the test section, and the blunt-nose was positioned $0.1 \mathrm{~m}$ downstream from the hypersonic nozzle exit. The angle of attack was maintained at $0^{\circ}$. This model had an injection system inside itself, which was composed of one/two sonic nozzle(s) and a plenum chamber. The sonic nozzle was a brass pipe which had a circular cross-section with outerdiameter $3.0 \mathrm{~mm}$ and inner-diameter $D=1.5 \mathrm{~mm}$. One/two holes of 3-mm diameter was/were made on the model surface to flush-mount the sonic nozzle(s). Hence, the cross-section of the brass pipe was exposed to the model surface (see 


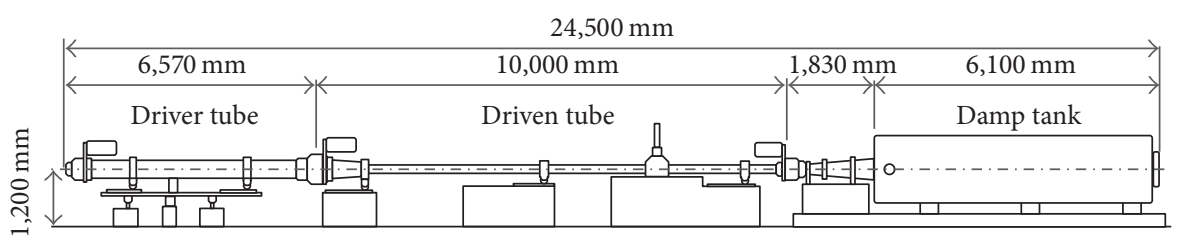

FIGURE 2: Dimensions of the shock tunnel of Nagoya University.

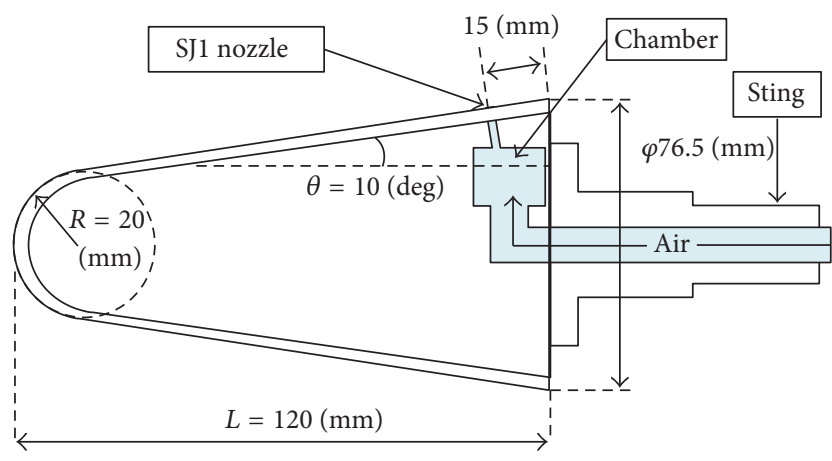

FIGURE 3: Dimensions of the test model.

Figure 5), and any paints were not applied onto this region. Air pressure was supplied from an external pressure chamber and controlled by a pressure regulator. The injection and the measurement were triggered by a signal at a TransistorTransistor Logic (TTL) level which is generated when the shock tunnel starts. The solenoid valve was driven by a DC power-supply which was switched by solid-state relay. This system was employed to retain the initial vacuum condition in the damp tank.

A two-dimensional coordinate system along the model surface was defined as shown in Figure 5. The origin of the coordinate system was set at injection hole SJ1, which was $15 \mathrm{~mm}$ upstream from the trailing edge of the model. The angle of rotation, $\varphi$, was defined to express a line that passed through an injector and the apex of the cone. The $x$-axis was positioned at $\varphi=0^{\circ}$. The relative positions of the two injection holes shown in Figure 5 were controlled as a test parameter; the locus of another injection hole (SJ2) was changed to control the distance between two injection holes, while SJ1 was fixed. Two different configurations of dual injection were tested. The first one was the "tandem" configuration, where the two injection holes were aligned streamwise. The location of SJ2 was changed at three levels, $S_{x}=3.36 D, 6.72 D$, and 10.1D. The second was the "parallel" configuration, where the injectors were aligned in a normal direction to the stream. The spacing between the two injection holes was changed at three levels, $S_{Y}=4 D, 8 D$, and $12 D$. Thus, the second injection hole (SJ2) positions were $\varphi=9.6,19.2$, and $28.8^{\circ}$, respectively. All of the jets were injected through the sonic nozzle at a constant total pressure, $P_{0 J}=0.71 \mathrm{MPa}$. NPR $=P_{0 J} / P_{\infty}$ was retained at NPR $=1.01 \times 10^{3}$.

Coaxial-thermocouples were flush-mounted on the model surface for the reference in the noninjection case.
They were aligned on the $x$-axis and located at $X / D=$ $-50,-43.3,-36.7,-30,-23.3,-16.7,-3.3$, and 3.3 .

2.3. Temperature Sensitive Paint. Temperature sensitive paint (TSP) is a temperature-measurement technique based on image sensing of temperature quenching of the paint [14]. Because luminescence from the paint is detected by a complementary metal oxide semiconductor (CMOS) camera, global information of surface temperature of the model can be obtained with a fine spatial resolution. The paint employed in this study consisted of Ruphen as the luminophore, polyacrylic acid as the binder, and ethanol as the solvent. Ruphen is a type of Ruthenium complex and a type of typical temperature sensitive luminophore used for TSP [15].

TSP was sprayed over a white polyurethane resin (PUR) layer, which was applied onto the model surface, except for the circular region with a diameter of $2 D$ (i.e., $3 \mathrm{~mm}$ ) around each injector hole. This base coating provided a low thermal effusivity, $\sqrt{\rho c k}$, which was necessary for measurements with TSP to assume one-dimensional thermal conduction in the wall, for a short duration of test time. The thermal properties of PUR at room temperature were measured by differential scanning calorimetry (specific heat capacity, $c_{b}$ ) and the periodic heating method (thermal diffusivity, $\alpha_{b}$ ). The values were determined as $\rho_{b}=1.02 \times 10^{3} \mathrm{~kg} / \mathrm{m}^{3} \pm 5 \%, c_{b}=$ $1.45 \mathrm{~kJ} / \mathrm{kg} \mathrm{K} \pm 6 \%$, and $\alpha_{b}=1.36 \times 10^{-7} \mathrm{~m} / \mathrm{s}^{2} \pm 5 \%$. Thus, the uncertainty in the Stanton number calculation was $8.2 \%$. The thickness of the base coat was controlled to be thicker than $0.1 \mathrm{~mm}$. In addition, the white base coating also enhanced signal-to-noise ratio (SNR) on the detection of luminescence emitted from the paint.

The TSP measurement system is also shown in Figure 4. The TSP layer was excited by a blue, 24-W LED, which had a wavelength of $450 \pm 50 \mathrm{~nm}$. This LED was fixed at $|O A|=0.3 \mathrm{~m}$ inside the damp tank. The luminescence emitted from the TSP layer was captured by a high-speed camera (FASTCAM-1024PCI, Photron), which was fixed at $|O B|=1.1 \mathrm{~m}$ outside the test section, with $512 \times 512$ pixels, a 3000 -fps frame rate, and a 1/3,000 s exposure time. A 580-nm long-pass filter was inserted in front of the camera lens. Spatial resolution of the TSP measurements was approximately $0.35 \mathrm{~mm} /$ pixel at $\varphi=0^{\circ}$.

2.4. Temperature- and Pressure-Sensitivity of the Paint. Temperature- and pressure-sensitivity of the paint were investigated in advance. A schematic of the calibration system is shown in Figure 6. TSP was applied over a piece of aluminum plate coated with white PUR paint. The specimen 


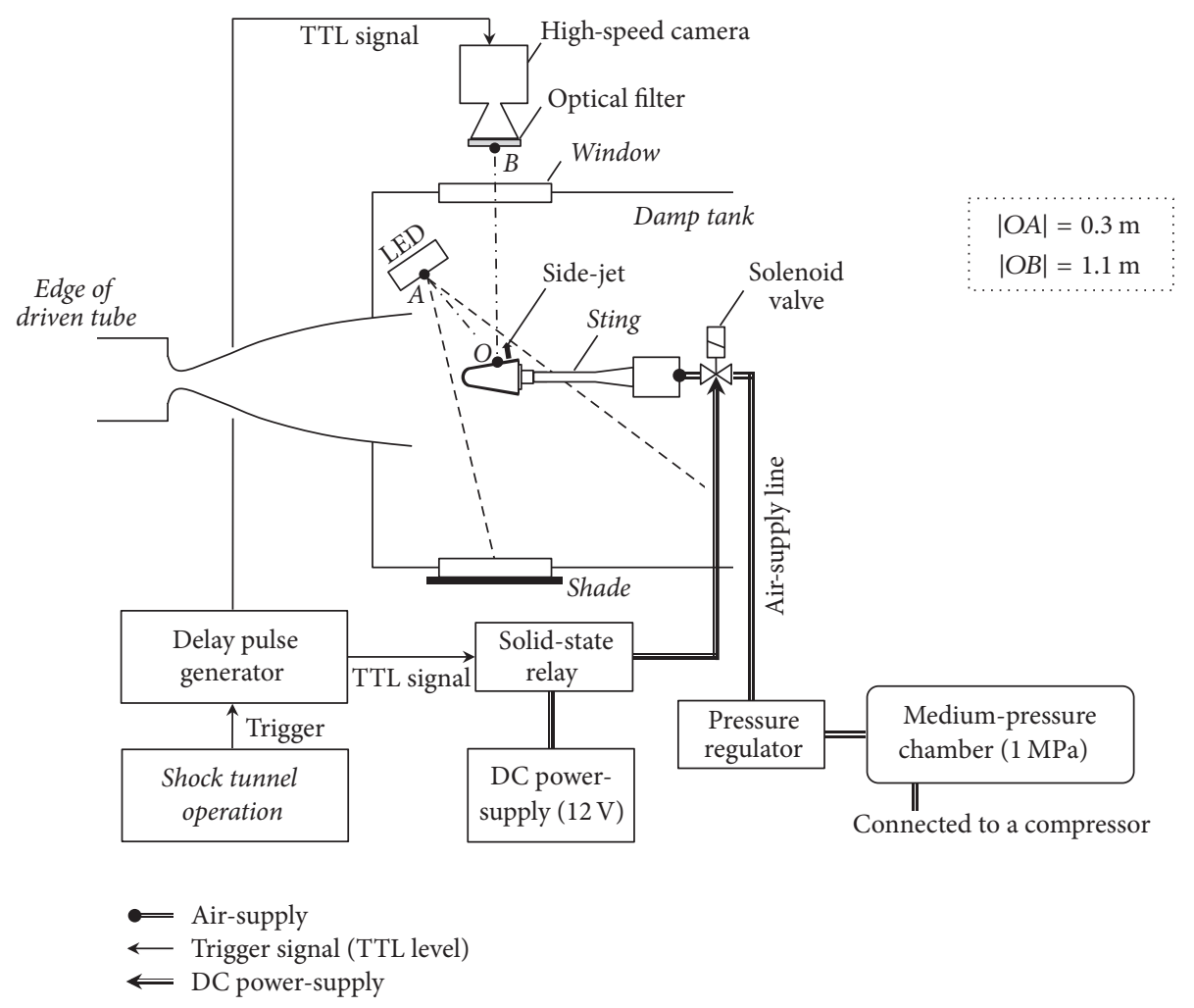

FIGURE 4: Schematic of the experimental system.

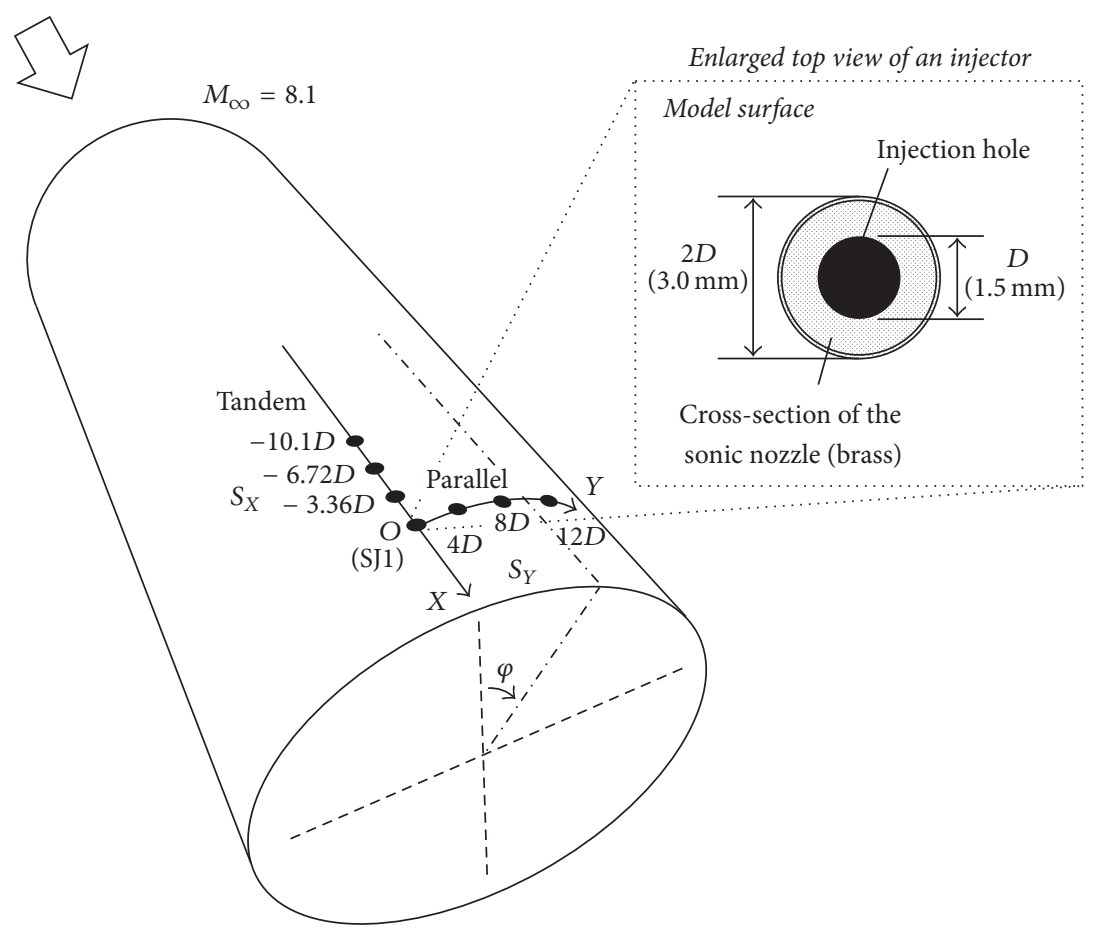

FIGURE 5: Injector arrangements and coordinate system along the model surface. 


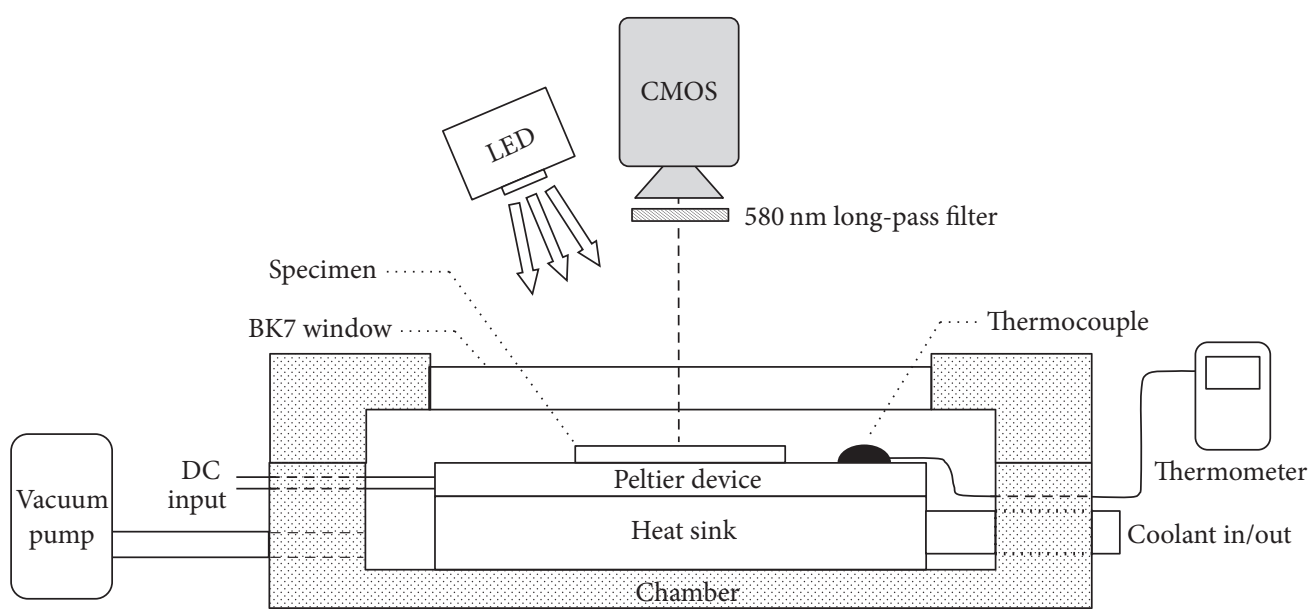

FIgURE 6: Calibration chamber of temperature sensitive paint.

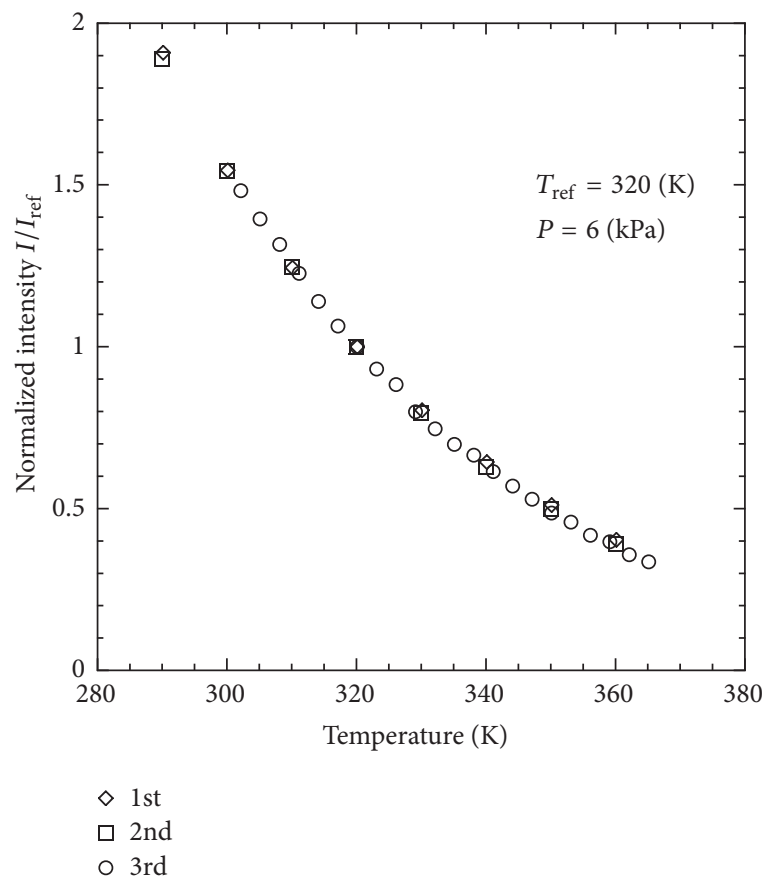

FIgURE 7: Temperature-sensitivity of the temperature sensitive paint in a vacuum $(6 \mathrm{kPa})$.

was placed onto a Peltier device, which was mounted on a heat sink in the chamber. The surface temperature on the Peltier device was controlled by a DC power-supply and monitored by a type-K thermocouple. The TSP was excited by the blue LED through the window, and the luminescence emitted from the paint was detected by the CMOS camera with the optical filter. The pressure could also be controlled from a vacuum $(\sim 6 \mathrm{kPa})$ to $1 \mathrm{~atm}$ by using a vacuum pump.

The temperature-sensitivity of the paint is shown in Figure 7. Calibrations were performed three times in the vacuum condition with individual specimens to confirm repeatability of the temperature-sensitivity. Each specimen was prepared with individual paints, which had the same proportion of the constituents. Each curve showed good repeatability. The temperature-sensitivity was $3.2 \% / \mathrm{K}$ at $300 \mathrm{~K}$, and it decreased as the temperature was increased. All data obtained by means of TSP was converted from a normalized-intensity map into surface temperature distribution on the model. A calibration at $1 \mathrm{~atm}$ was also conducted to investigate the pressuresensitivity of the paint. It is shown in Figure 8 that the largest difference was observed at $305.15 \mathrm{~K}$, which involved $5.3 \%$ oxygen quenching, while the pressure difference was $95 \mathrm{kPa}$. In the wind tunnel tests, the static pressure of the uniform flow was $377 \mathrm{~Pa}$, and the stagnation pressure was $32.1 \mathrm{kPa}$. Thus, the pressure differential between static and pitot pressure was $31.7 \mathrm{kPa}$. This differential pressure induced $1.8 \%$ oxygen quenching at the stagnation point. Therefore, the temperature was overestimated by $0.6 \mathrm{~K}$ at the 


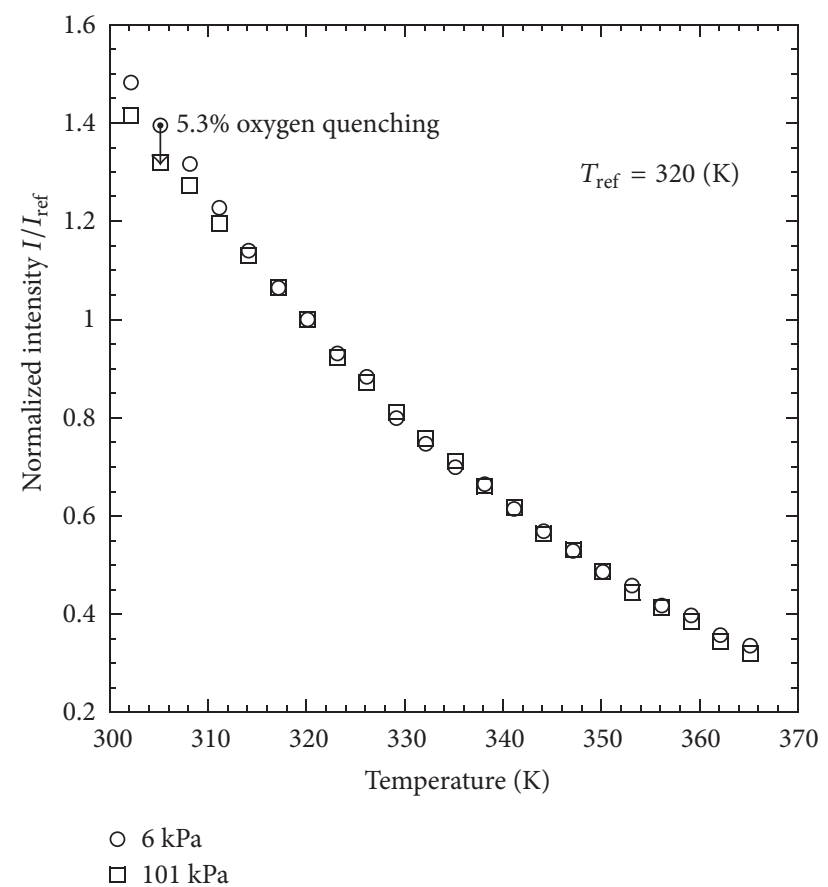

FIGURE 8: Pressure-sensitivity of temperature sensitive paint.

maximum due to the pressure-sensitivity. This error was only $0.7 \%$ of the temperature increment of the TSP layer at the stagnation point, which was approximately $80 \mathrm{~K}$. Hence, the pressure-sensitivity of the TSP had a negligible impact on the temperature-sensitivity.

2.5. Stanton Number Calculation. The convective heat transfer rate on the model surface can be calculated by using (1). It is based on one-dimensional heat conduction in a semiinfinite medium, including a linear approximation proposed by Cook and Felderman [16]. According to this equation, surface heat transfer depends on the thermal properties of the base material and the history of temperature rise. Therefore, the heat flux and Stanton number, which is calculated from TSP data, may include an error due to the uncertainty of the thermal properties.

$$
\dot{q}\left(t_{n}\right)=2 \sqrt{\frac{\rho c k}{\pi}} \sum_{i=1}^{n} \frac{T\left(t_{i}\right)-T\left(t_{i-1}\right)}{\left(t_{n}-t_{i}\right)^{1 / 2}+\left(t_{n}-t_{i-1}\right)^{1 / 2}} .
$$

The heat transfer rate is described in terms of the Stanton number, which was proposed by Abgrall et al. [17]. The definition of Stanton number is as shown below:

$$
\mathrm{St}=\frac{\dot{q}_{w}}{\rho_{\infty} U_{\infty} c_{p, \text { air }}\left(T_{0}-T_{w}\right)} \text {. }
$$

2.6. Validation of TSP Data. St distribution on the $x$-axis in the case of no injection is shown in Figure 9, where the closed circle represents the thermocouple data and the curves represent the TSP data. The mean value of St measured by TSP is about $8 \times 10^{-3}$ at $X / D=-50$ and tends to decrease

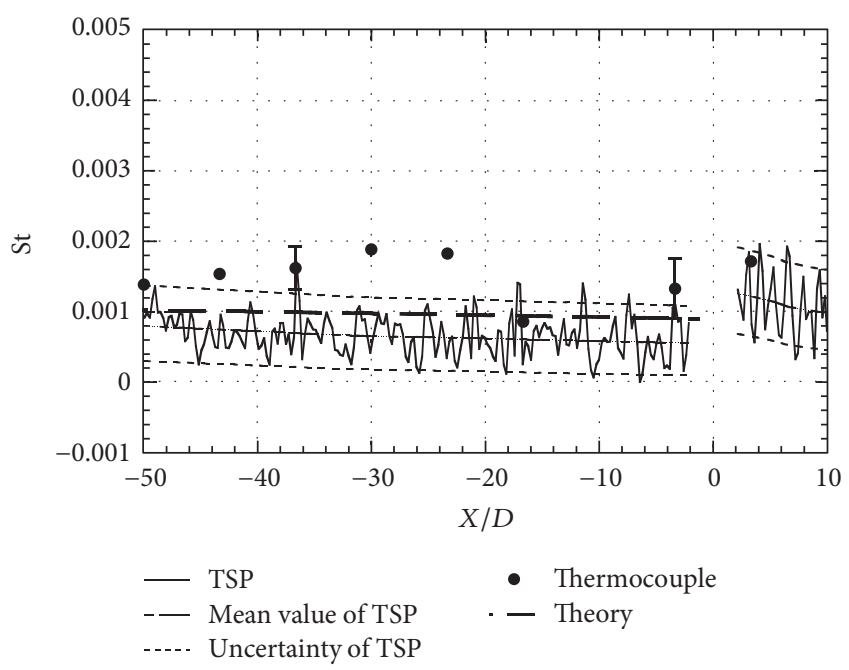

FIGURE 9: Stanton number profile on $x$-axis obtained by TSP, thermocouples, and Lees' equation (as a reference) under noinjection condition at $t=30 \mathrm{~ms}$. The upper and lower broken lines indicate the range of uncertainty of the TSP measurements. The error bar of thermocouple data indicates $\pm 1 \sigma$ calculated with data obtained in two runs.

gradually according to $X / D$. The St differential between the value at $X / D=-50$ and -3 was $\Delta \mathrm{St}=-2 \times 10^{-4}$ in terms of the mean value.

A reference St, based on Lees' equation [18], is also shown as a broken line. The theory gives heat flux distribution on a hemisphere-cone body as a ratio to stagnation value. The heat flux at the stagnation point is calculated by using the Fay-Riddell equation [19]. The wall temperature, which is necessary to calculate St, was calculated using (3). This equation was derived from one-dimensional heat conduction on a semi-infinite wall, with a constant heat flux input. Then $\mathrm{St}$ is calculated by using (2). The value predicted by the theory is consistent with the TSP data in the range of uncertainty.

The standard deviation of the TSP data was $4 \times 10^{-4}$, in terms of St, which is attributable mainly to the camera noise. The SNR was approximately 2 against the minimum St at St $=8 \times 10^{-4}$ in a no-injection condition, whereas it reached approximately 30 against the maximum St at $S t=1.5 \times 10^{-2}$, in a case with injections. Error induced by the curvature of the model was also investigated experimentally. It was uniquely determined by the local luminous intensity of the TSP layer. On the model, the error increased gradually as $\varphi$ increased and reached double at $\varphi=60^{\circ}$. Slight movement of the wind tunnel model due to aerodynamic forces also induced another measurement error. In this study, the movement of the projected image of the model on the CMOS sensor was less than one pixel of the CMOS sensor. Even if the model image moves by one pixel, the relative error in terms of St is less than St $=2 \times 10^{-5}$, which is much smaller than the camera noise. By contrast, the model movement caused a significant error in the vicinity of injection holes, because the detected intensity was affected by the hole displacement. Therefore, St data in a circular region with a diameter of 
$3 D$ (i.e., $4.5 \mathrm{~mm}$ ) around injector holes were intentionally omitted from all the plots and maps. Another error factor of the TSP data was the uncertainty of the thermal properties of the PUR. As shown in (1) and (2), St is proportional to the thermal effusivity; thus, its uncertainty is added to the uncertainty of St directly. The range of uncertainty of St on the TSP data, as shown in Figure 9 by broken lines, included $\pm 1 \sigma$ camera noise, uncertainty of the thermal effusivity of PUR, and dispersion of test flow conditions.

The thermocouple measurement was conducted over six runs under the no-injection condition, because number of available thermocouples for a run was limited to only two thermocouples. The scattered data of the thermocouple was distributed along the upper limit of uncertainty of the TSP data and is consistent with the TSP data in the range of uncertainty. The data at $X / D=-36.7$ and -3.3 were measured twice and thus each plot has an error bar. The standard deviation was $4 \times 10^{-4}$ at $X / D=-3.3$ and $3 \times 10^{-4}$ at $X / D=-36.7$. Since the error was larger than the differential $\Delta S t$, the decreasing tendency of TSP data was not detected by the thermocouples. The dispersion of the thermocouple data was caused by the following two factors: (1) dispersion of the test flow conditions which included a 9\% scattering in terms of St at the stagnation point and (2) low SNR on the thermocouple measurement. Temperature increment in the short duration $(30 \mathrm{~ms})$ is less than $1 \mathrm{~K}$.

$$
T_{w}(t)=T_{i}+\frac{2 \dot{q}_{w} \sqrt{t}}{\sqrt{\pi} \sqrt{\rho c k}} .
$$

\section{Result and Discussion}

3.1. Single Injection. The Schlieren photograph in the case of the single injection is shown in Figure 10(a). The sidejet was injected at the point $a, X / D=0$. The flow field includes a barrel shock, bow shock, and separation shock. The surface distribution of St is shown in Figure 10(b). Three characteristic regions, $\mathrm{A}, \mathrm{B}$, and $\mathrm{C}$, can be defined in terms of heating patterns as illustrated in Figure 10(c). In region $A$, the primary heat transfer increase takes place. According to the previous study [6], region A corresponds to the reattachment line of the primary horseshoe vortex. Point $b$ is defined as the point of maximum $\mathrm{St}$ on the $x$-axis in region $\mathrm{A}$. The St profile on the $x$-axis is shown in Figure $10(\mathrm{~d})$. Point $b$ is located at $X / D=-1.6$, immediately upstream of the sidejet and just behind the bow shock. Upstream of point $b$, a small hump appears at point $c$, which is at $X / D=-6.0$. Point $c$ is the center point of region B shown in Figure 10(c). Region $B$, which is included in the separated region of the boundary layer as shown in Figure 10(a), corresponded to the reattachment line of the secondary horseshoe vortex in the separation region, that is, separation vortex. In Figure 10(d), for $-13<X / D<-10$, St is lower than the level in the undisturbed area. Region $C$ represents the area of the low thermal load and corresponds to the separation line of boundary layer. Point $d$ is defined as the point of minimum St. The heating pattern is similar to that obtained by Powrie et al. [6] under $M_{\infty}=6.69$ and $\operatorname{Re}_{\infty}=4.27 \times 10^{6} \mathrm{~m}^{-1}$.
3.2. Dual Injection in Tandem Configuration. Schlieren photographs in the tandem configuration are shown in Figure 11. $S_{x}$ is defined as the distance between two jets, SJ1 and SJ2. When comparing Figure 10(a) in the single injection case with Figure 11(a) in the tandem configuration, the barrel shock of SJ2 is much smaller in the tandem configuration than in the single injection case. The size of the barrel shock of SJ2 increases with $S_{x}$. On the other hand, the barrel shock of SJ1 is larger than in the single injection case, while its size increases with $S_{x}$. The expansion of SJ1 is influenced by the presence of SJ2, since the interaction between the main stream and SJ1 is disturbed in the wake of SJ2. The barrel shock of SJ1 appears in the space outside the wake of SJ2. Because the region of influence of SJ2 expands downstream, the interaction between the main stream and SJ1 takes place at a higher level above the boundary of the wake of SJ2 when $S_{x}$ is increased.

Surface St distributions in the tandem configuration are shown in Figures 12-14. The heating patterns in the tandem configuration are basically similar to those in the single injection case and the three characteristic regions $(A, B$, and C) and the characteristic points $(b, c, d)$ can be defined.

The most remarkable feature in the tandem configuration was the reduction of the separation area. As shown in Figure 12(c), the point $d^{\prime}$, which corresponded to the separation point in the tandem configuration, was closer to the exit of SJ2 than the point $d$ in the single injection case. Separation length, $l_{\text {sep }}$, is defined here as the length between point $d$ (or $d^{\prime}$ ) and the exit of the SJ2. $l_{\text {sep }} / D$ is around 10 both in the tandem configuration and in the single injection case. In the case of the tandem configurations, for $S_{x}=3.36 D$ and $6.72 D$, the difference between $d$ and $d^{\prime}$, $\left|d-d^{\prime}\right|$, is around $15 \%$ of the separation length in the single injection case. By contrast, a smaller difference was observed for $S_{x}=10.1 D$. From the viewpoint of application in an RCS, the separation of the boundary layer enhances the thrust, because the separation region generally indicates a moderate pressure rise, which contributes to the amplification of the thrust as reported in previous studies $[4,5]$. Thus the reduced separation area in the tandem configuration could diminish the thrust amplification.

The St profile, along the $x$-axis for $S_{x}=3.36 D$, is shown in Figure 12(c). The maximum value of $\mathrm{St}$ in the tandem configuration, at point $b^{\prime}$, is smaller than in the single injection case, by $23 \%$. Figure 13(c) shows the St profile for $S_{x}=6.72 D$ and Figure 14(c) for $S_{x}=10.1 D$. In those cases, the maximum values of St were similar to those in the single injection cases.

Another feature of the tandem configuration is the shape of region A. $R_{\mathrm{A}}$ is defined as the distance from the $x$-axis to the ridgeline of region $\mathrm{A}$. Figure 15 shows $R_{\mathrm{A}}$ in both the single and tandem configurations. $R_{\mathrm{A}}$ increases monotonically with $X$. In the single injection cases, each $X / D$ $R_{\mathrm{A}}$ curve approximates a quadratic curve. In the tandem configurations, a discontinuous point appears near $X=0$, which corresponds to the locus of SJ1. Downstream of the discontinuous point, the rate of increase of $R_{\mathrm{A}}$ increases. This feature is due to the interference of a horseshoe vortex, 


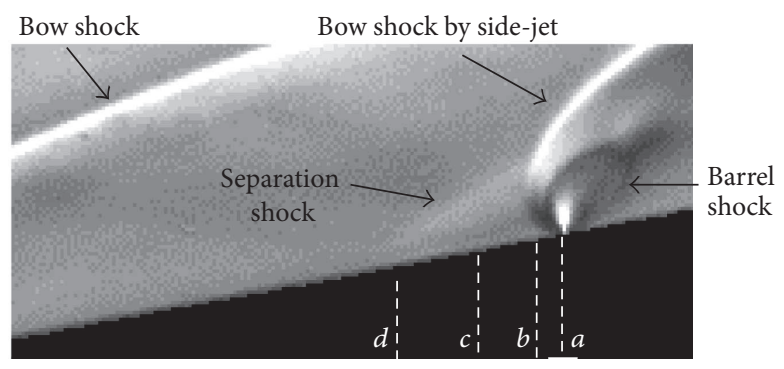

(a)

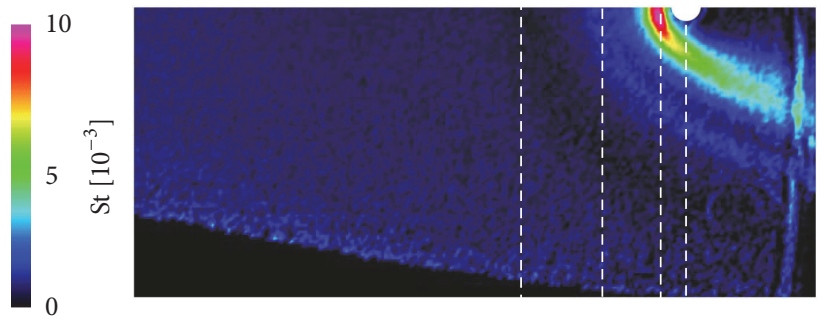

(b)

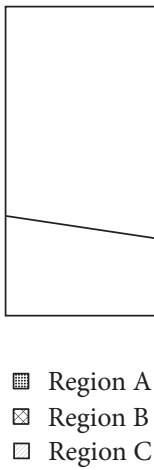

冈 Region $\mathrm{B}$

$\square$ Region $\mathrm{C}$

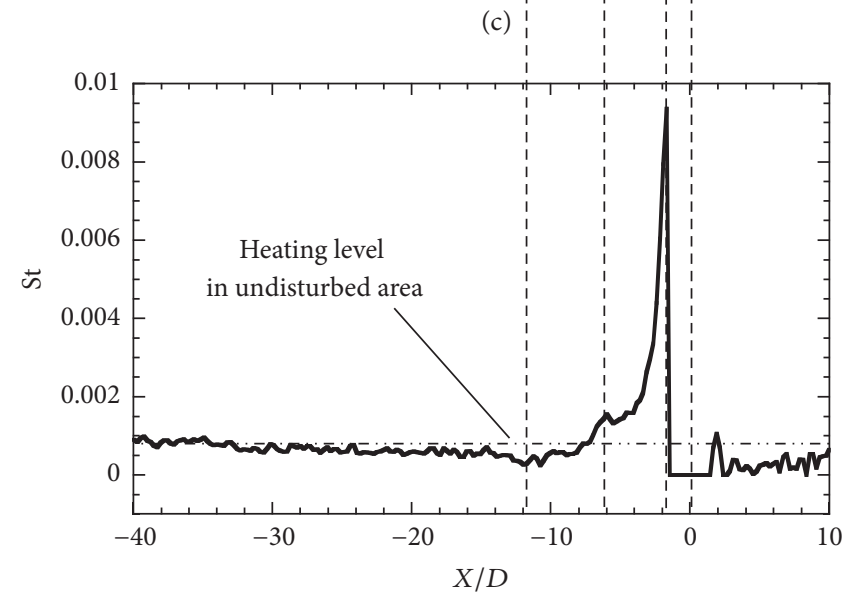

(d)

FIGURE 10: St distribution in the case of single-jet injection. (a) Schlieren photograph with single injection at SJ1. (Point $a$ : locus of injection hole, point $b$ : locus with peak Stanton number, point $c$ : peak by separation vortex, and point $d$ : separation point.) (b) Surface distribution of St measured by TSP. (c) A schematic of the heating patterns and (d) a one-dimensional profile of St on the $x$-axis. 


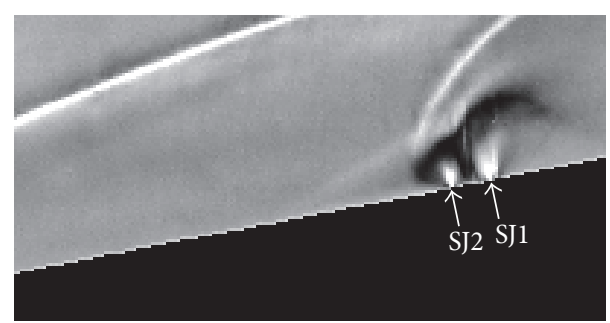

(a)

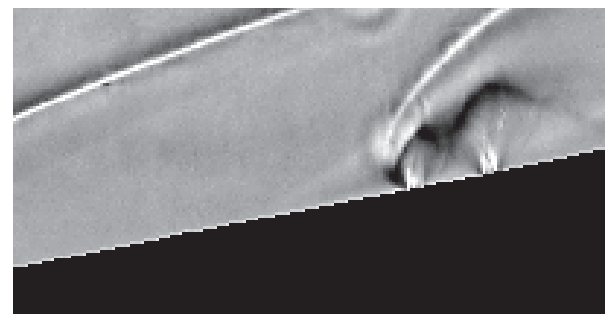

(b)

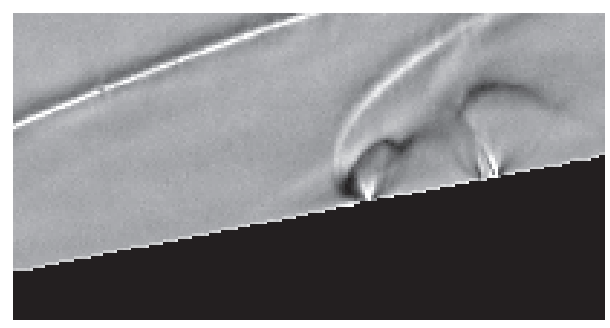

(c)

FIGURE 11: Schlieren photographs for the tandem dual injections at (a) $S_{x}=3.36 D$, (b) $S_{x}=6.72 \mathrm{D}$, and (c) $S_{x}=10.1 \mathrm{D}$.

originating in front of SJ1, on the horseshoe vortex from SJ2. The existence of the horseshoe vortex around the rear sidejet, which corresponds to SJ1 in this study, has been reported by Lee [9]. Moreover, in the tandem configurations for $S_{x}=$ $6.72 \mathrm{D}$ and $10.1 D$, the region A upstream of the discontinuous point is thinner than in the single injection case, while there is little difference between two configurations for $S_{x}=3.36 \mathrm{D}$. This is because of the reduction in the expansion of SJ2 shown in Figure 11. For $S_{x}=3.36 D$, the barrel shocks of SJ1 and SJ2 merged, to behave as a single obstacle rather than two individual shocks. This is why the surface heating pattern in this case is similar to the single injection case. Furthermore, as indicated in Figures 13(b) and 14(b), an additional heating area appeared in region $\mathrm{A}$. A moderate heat transfer increase, approximately at $\mathrm{St}=3 \times 10^{-3}$, takes place in this area. This feature does not appear for $S_{x}=$ 3.36D. The heat transfer increase area was formed along the reattachment of the horseshoe vortex from SJ1, as well as in region $\mathrm{A}$.

3.3. Dual Injection in Parallel Configuration. Figures 16(a)-16(c) show the surface St distribution for the dual injections in the parallel configuration. Regions A to C can be defined as in the case of single injection. The geometries of the regions vary significantly depending on the injection hole spacing $S_{Y}$. In Figure 16(a), for $S_{Y}=4 D$, the primary heating area, region $\mathrm{A}$, represents an arch geometry, which is similar to the single injection case. The boundary of region $\mathrm{B}$ is unclear and indicated as a broken line. Further upstream, St drops slightly in region $C$ on the boundary layer separation. In contrast, in Figure 16(c), for $S_{Y}=12 D$, region $\mathrm{A}$ is formed around each jet. All region $\mathrm{A}$ shapes showed an asymmetric arch. Two arches of region $\mathrm{B}$, each of which was generated outside of region $\mathrm{A}$, merge near the $x$-axis. Region $\mathrm{C}$ has a single-arch shape. St distribution for $S_{Y}=8 D$ is different from those for $S_{Y}=4 D$ and $12 D$. Although region $\mathrm{A}$ originated in front of each injector, they overlap in the middle of the injectors. Consequently, region A appears as a trident-shape, with a concave leading edge.

St profiles along the $x$-axis and on an axis passing through an injector are also shown in Figures 16(a)-16(c). Maximum values of St in the parallel configurations are smaller than in the single and tandem configurations. The reduction of heat transfer increase is most effective for $S_{Y}=8 D$. The maximum value was St $=4 \times 10^{-3}$, which is approximately $40 \%$ of the maximum in the single injection case. The locus of maximum St (point $b$ ) depends on $S_{Y}$. For $S_{Y}=4 D$ and $8 D$, point $b$ is on the $x$-axis. For $S_{Y}=12 D$, two points of the maximum St are located upstream of the jet exits, as in the single injection case.

The separation area is extended further upstream in the parallel configurations. The separation length is, at most, $l_{\text {sep }} / D=20.4$ for $S_{Y}=8 D$, which is $70 \%$ larger than in the single injector case. Even within the minimum extension for $S_{Y}=12 D$, the separation length is extended by $30 \%$ from the single injection case. In terms of applications for RCS, by using the parallel configurations, the thermal load on vehicle surface is diminished. However, it should be noted that the surface area influenced by the interaction is apparently enlarged, and thus thrust amplification can also be influenced more significantly.

These heating patterns can be explained in terms of the shock-shock interactions. Kawamura et al. [20, 21] investigated a two-dimensional shock system around two cylinders aligned normal to a supersonic stream. Three different types of shock system were generated, depending on the distance between the two cylinders, as illustrated in Figure 17. When the distance between the cylinders is large, as shown in Figure 17 (c), two shock waves cross at a single point between two cylinders. This regime is the so-called regular reflection (RR) [22]. At an intermediate distance, as shown in Figure 17(b), the incident angle of the shocks becomes larger so that the shock regime transits to Mach reflection (MR) [22], involving a triple point and a Mach stem. At a smaller distance, as shown in Figure 17(a), a single bow shock is generated in front of the two cylinders. Similar shock systems should be generated for the side-jet injection in parallel configurations. The geometry of region A reflects features of the shock system. For $S_{Y}=12 D$, the shock system is in the RR regime and for $S_{Y}=8 D$ in the MR regime, while the concave part of region A corresponds to the Mach stem. 


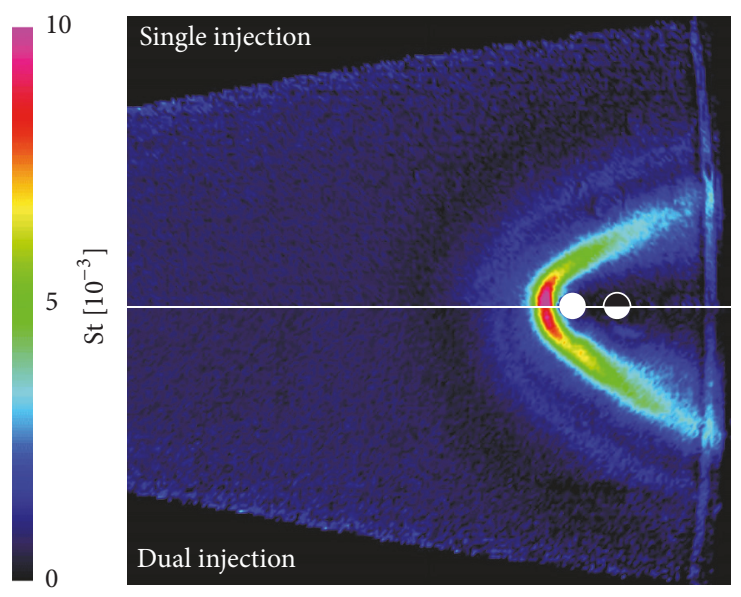

(a)

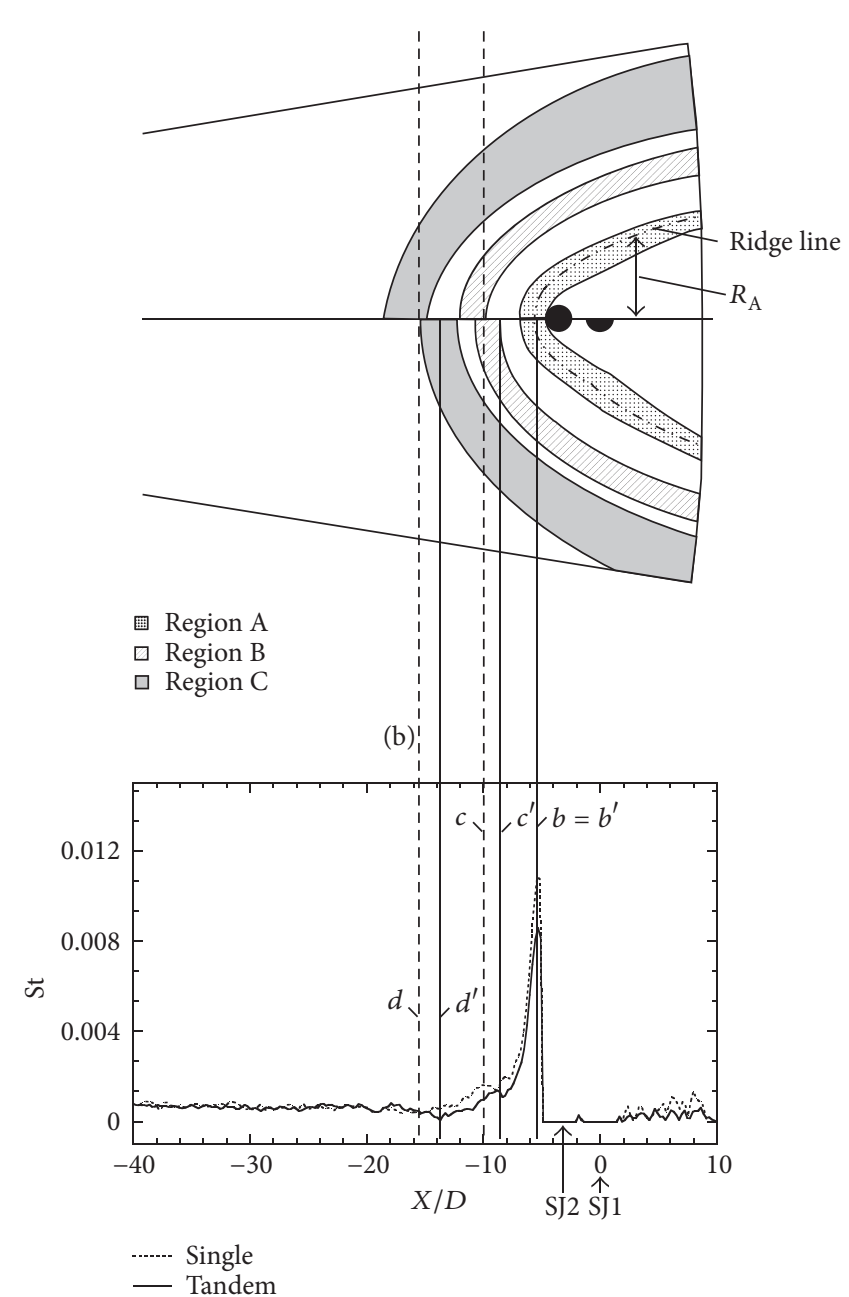

(c)

FIGURE 12: St distribution in the tandem configuration for $S_{x} / D=3.36$. (a) Surface distribution of Stanton number measured by TSP, (b) schematic of the heating patterns, and (c) one-dimensional profile of on the $x$-axis, with single and tandem dual injection at $S_{x}=3.36 D$. Point $b$, locus with peak Stanton number; point $c$, peak of separation vortex; and point $d$, separation point. Prime marks indicate the points with dual injections. The upper half of (a) and (b) displays a single injection case and the lower half a dual injection case. 


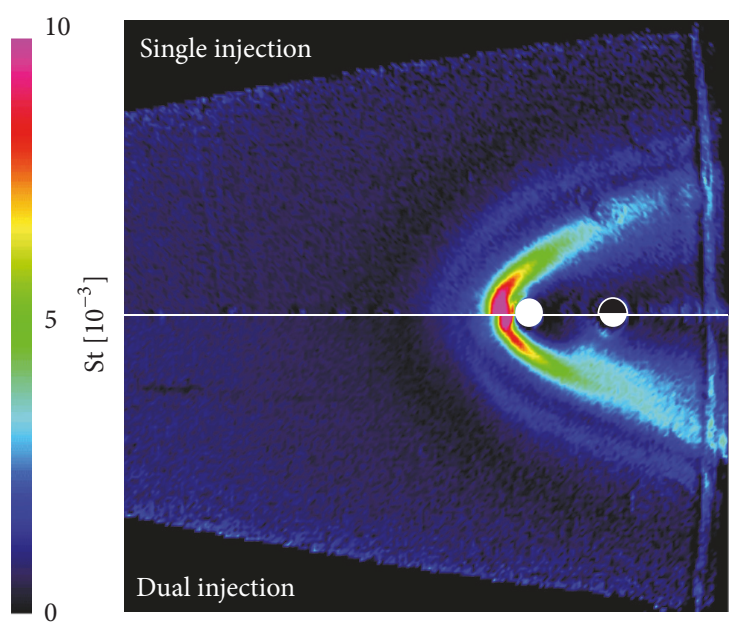

(a)

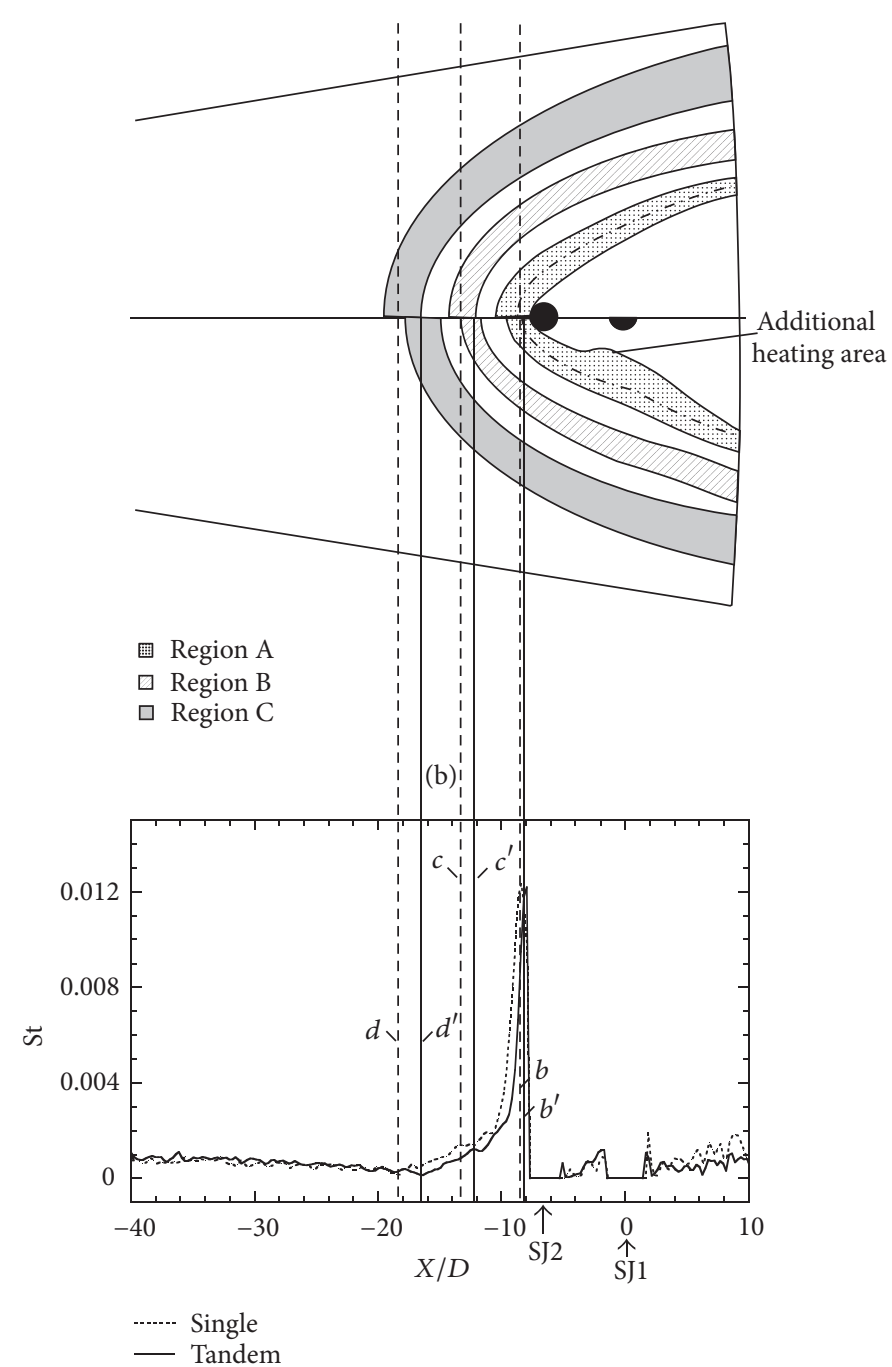

(c)

FIGURE 13: St distribution in the tandem configuration for $S_{x} / D=6.72$. (a) Surface distribution of Stanton number measured by TSP, (b) schematic of the heating patterns, and (c) one-dimensional profile of on the $x$-axis, with single and tandem dual injection at $S_{x}=6.72 D$. Point $b$, locus with peak; point $c$, peak of separation vortex; and point $d$, separation point. Prime marks indicate the points with dual injections. The upper half of (a) and (b) displays a single injection case and the lower half a dual injection case. 


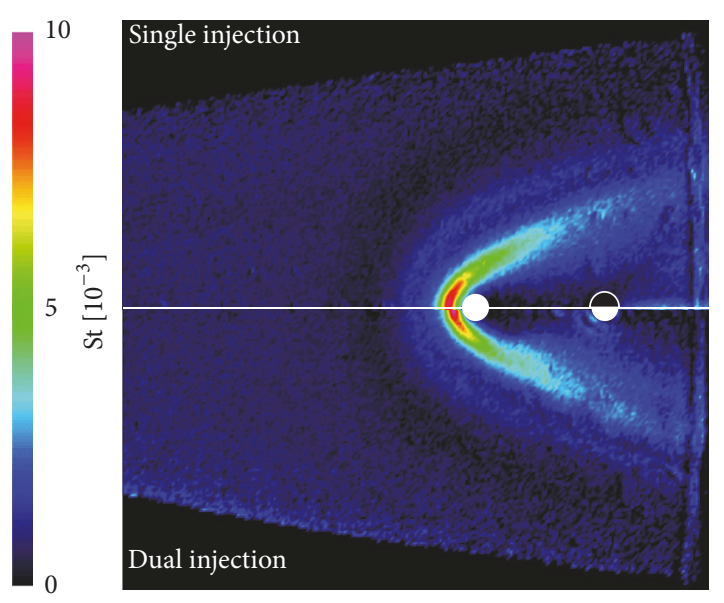

(a)

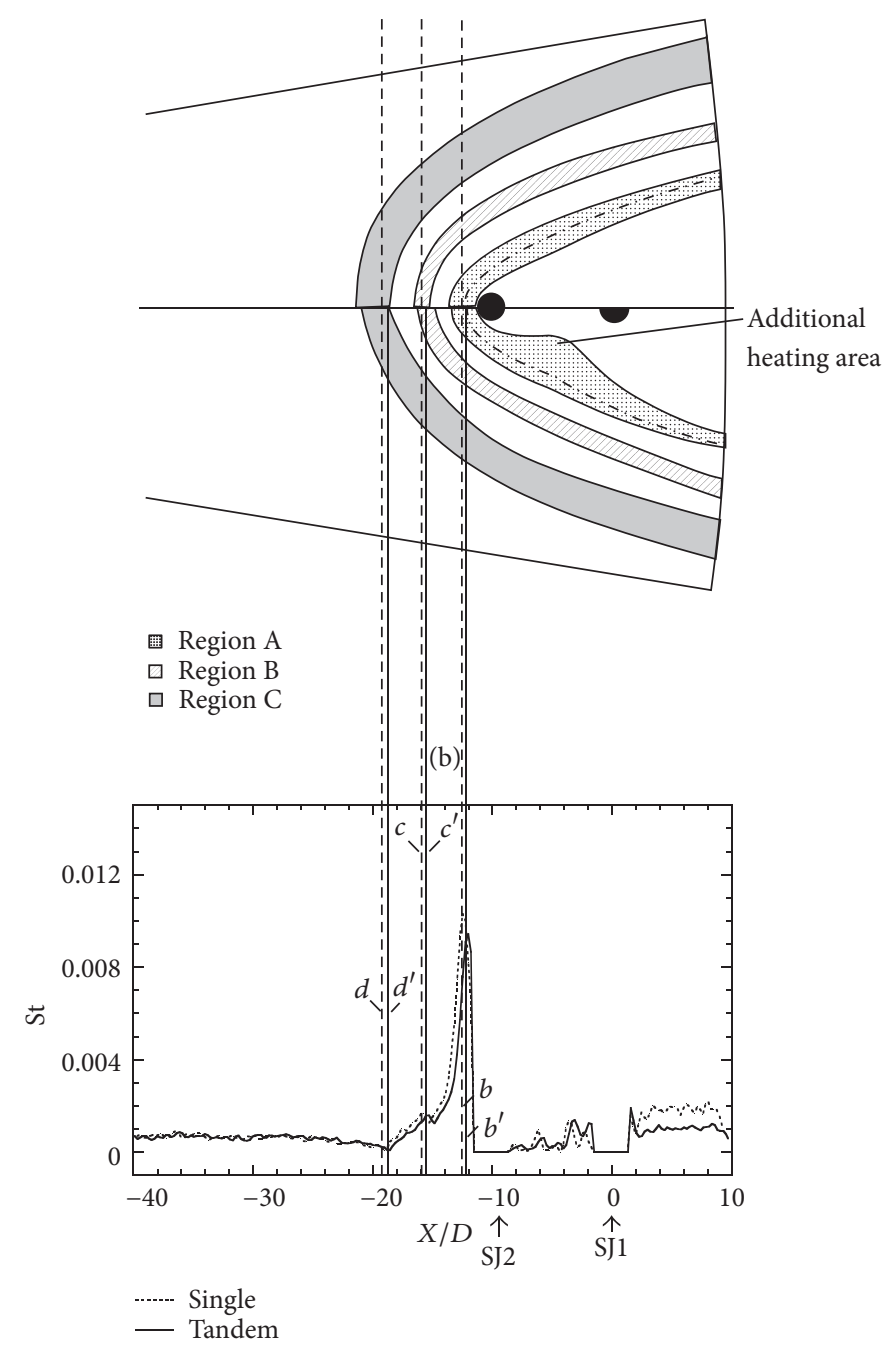

(c)

FIGURE 14: St distribution in tandem configuration for $S_{x} / D=10.1$. (a) Surface distribution of Stanton number measured by TSP, (b) a schematic of the heating patterns, and (c) one-dimensional profile of Stanton number on $x$-axis, with single and tandem dual injection at $S_{x}=10.1 D$. Point $b$, locus with peak Stanton number; point $c$, peak by separation vortex; point $d$, separation point. Prime indicates the points with dual injections. The upper part of (a) and (b) displays a single injection case and the lower half a dual injection case. 


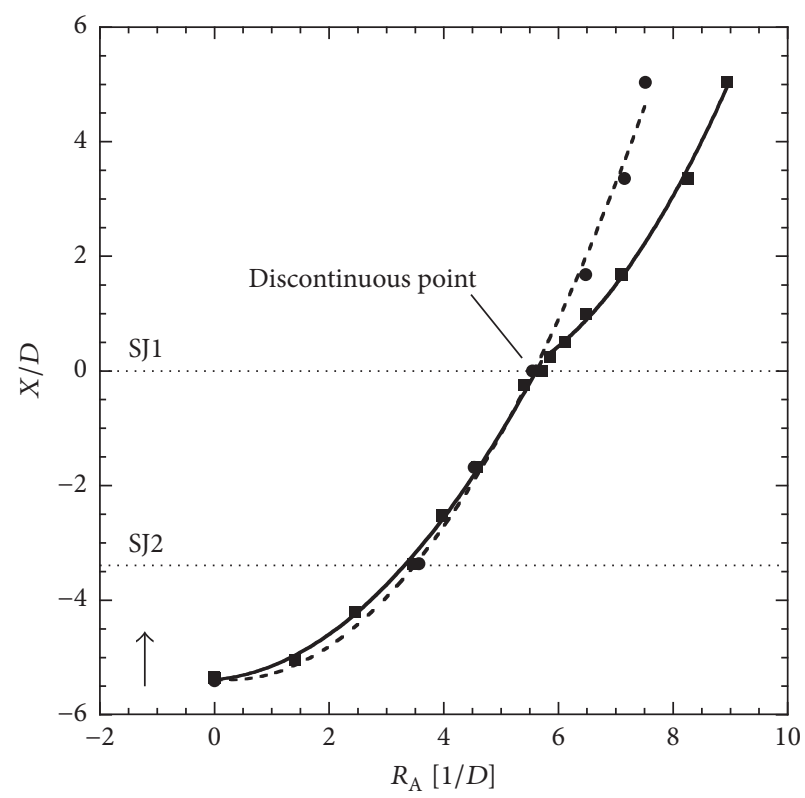

$\bullet$ Single $\rightarrow$ Tandem

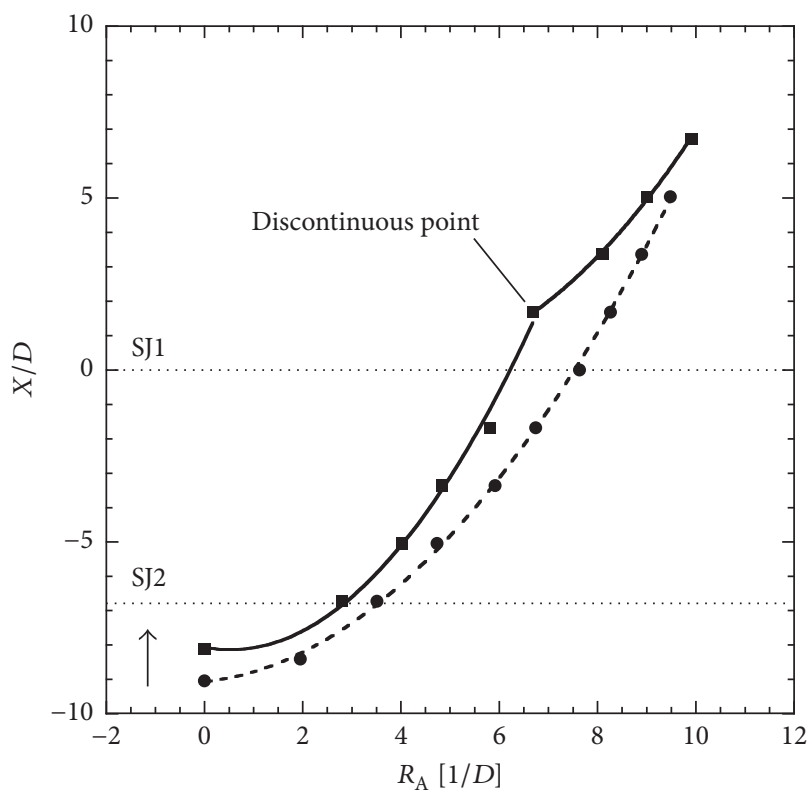

$\because$ Single

$\rightarrow$ Tandem

(a) $S_{x}=3.36 D$

(b) $S_{x}=6.72 D$

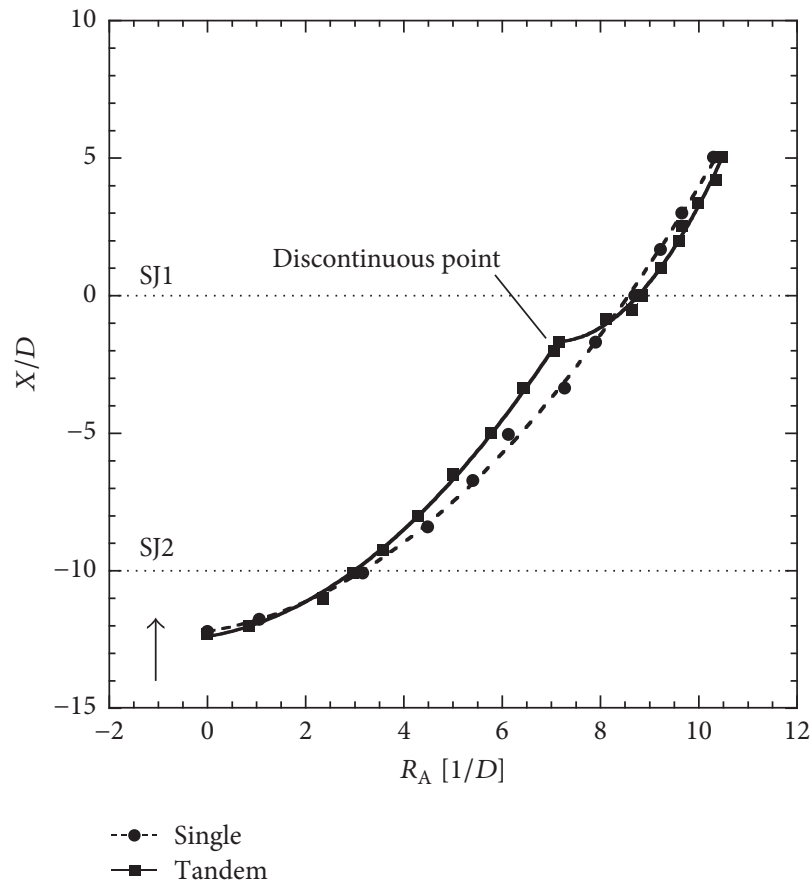

(c) $S_{x}=10.1 D$

Figure 15: Comparison of half width of region A induced by single and tandem dual injection(s) with each injection hole spacing. Plots indicate loci of maximum $S t$ in region $A$ on each $X / D$ position. $\uparrow$ indicates the freestream direction.

\section{Conclusion}

Aerodynamic heating induced by dual side-jets into hypersonic cross flow was investigated experimentally. Global heat flux distributions were represented in terms of Stanton number (St) by means of temperature sensitive paint (TSP), while the flow field was visualized by Schlieren photography. Two types of injector arrangements were employed; a tandem configuration with two injection holes aligned streamwise, and a parallel configuration with injector holes aligned 


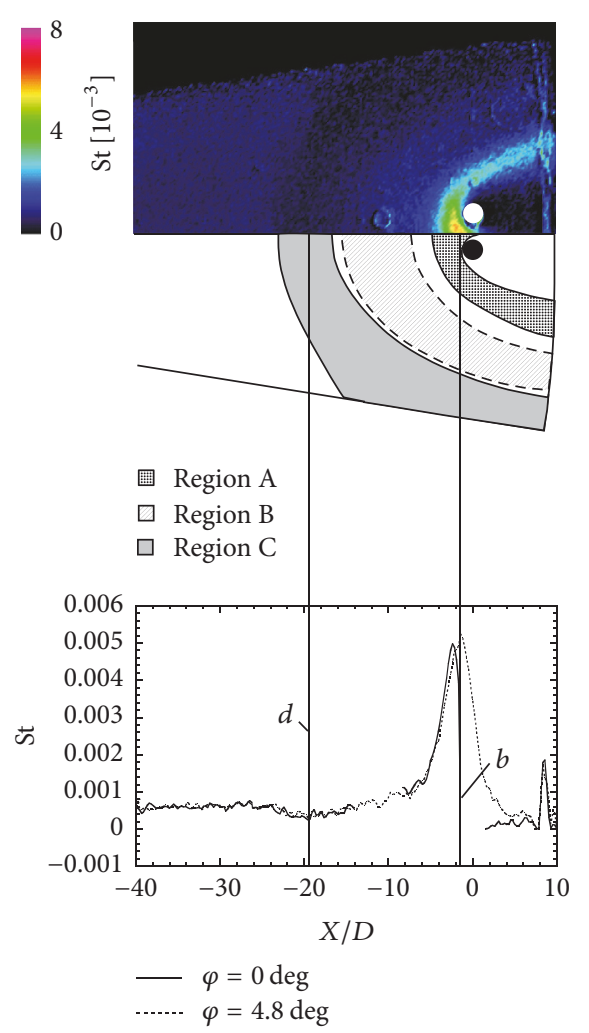

(a) $S_{Y}=4 D$

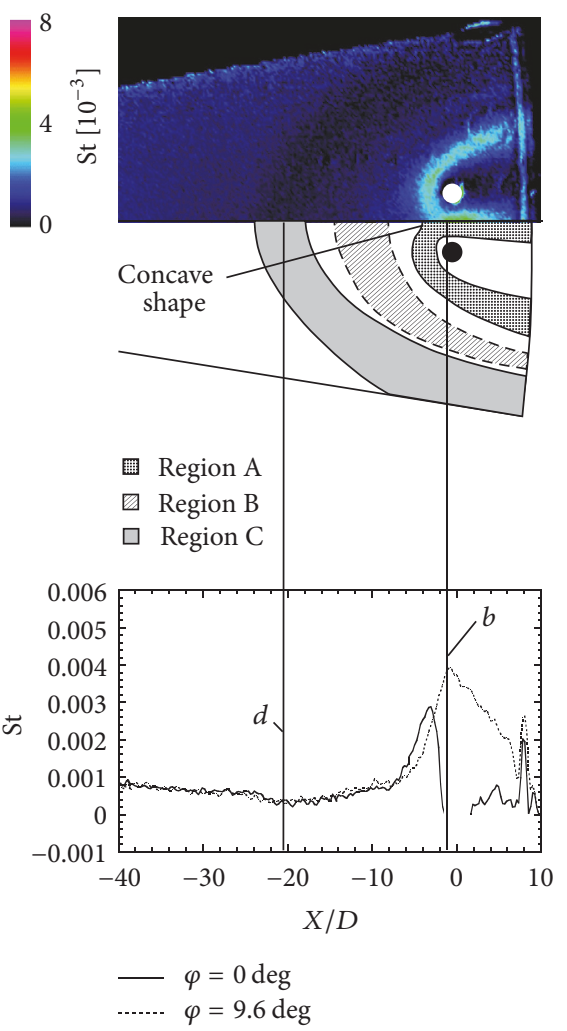

(b) $S_{Y}=8 D$

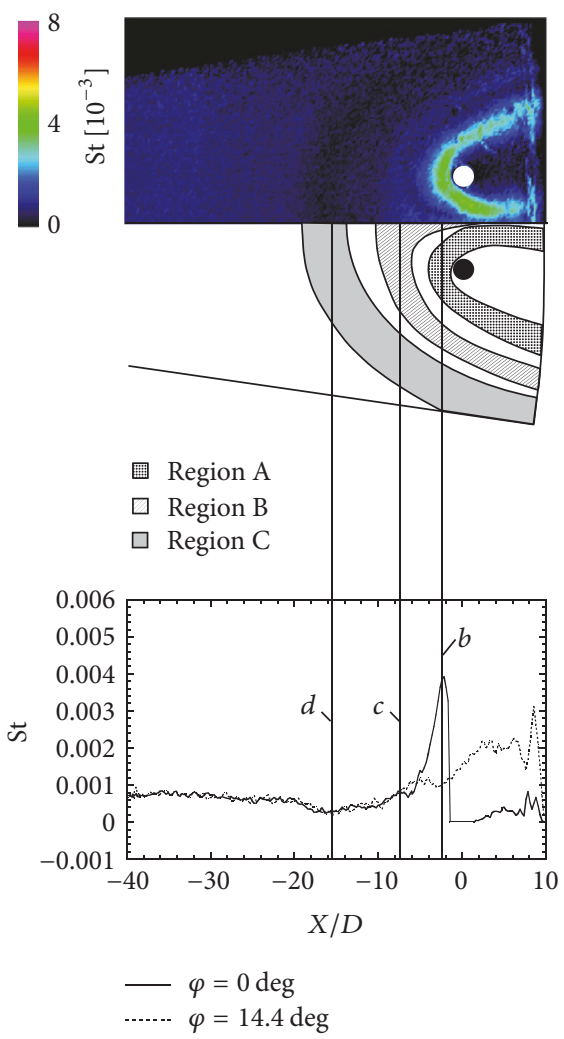

(c) $S_{Y}=12 \mathrm{D}$

FIGURE 16: Surface St distributions induced by parallel dual injections with each spacing of $S_{Y}$ (upper figures), schematics of heating patterns (middle figures), and one-dimensional St profile on the middle of dual injectors $(\varphi=4.8,9.6$, and $14.4 \mathrm{deg})$ and a line through $S J 1$ ( $\varphi=0$ deg) (lower figures). Unclear boundaries of region B are illustrated with broken lines in the middle figures. A section of the St profile at $S_{y}=4 D$ is not shown due to a measurement error. 


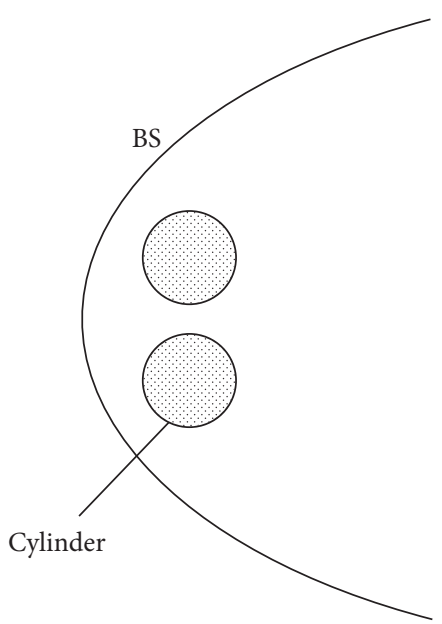

(a) $S_{Y}=4 D$

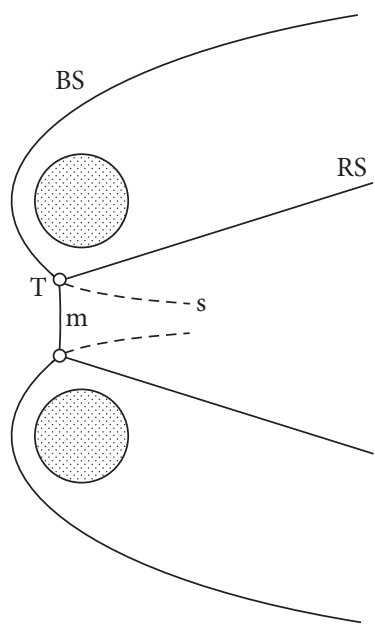

(b) $S_{Y}=8 D$

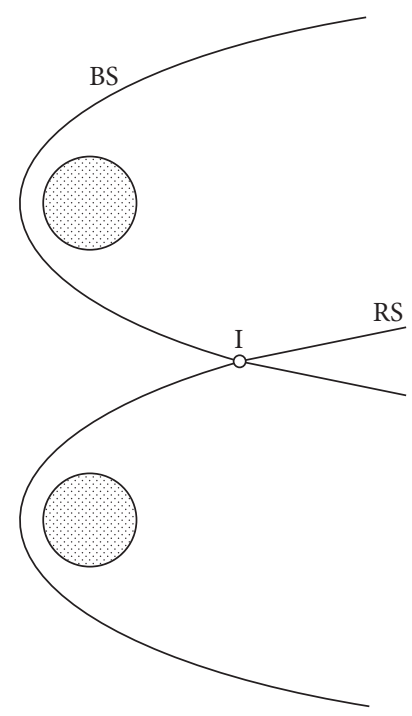

(c) $S_{Y}=12 D$

FIGURE 17: Three types of bow-shock interactions formed around cylinder arrays. BS, bow shock; RS, reflected shock; I, single interaction point; T, triple point; $\mathrm{m}$, Mach stem; s, slip line.

normal to the freestream. The influence of the distance between the dual injectors was investigated. The following results were obtained:

(1) For dual injections in the tandem configurations, St distributions were basically similar to the single injection cases. The surface heating patterns were slightly different from the single injection case, while the separation area upstream of the front side-jet was found to be diminished. Separation length was reduced by $15 \%$, at most, in comparison with single injection, and this effect was relaxed at larger injector spacing.

(2) For dual injections in the parallel configurations, the heat transfer increase was drastically diminished. The reduction was most effective at $S_{Y}=8 D$; the peak St was reduced by $60 \%$ from the single injection case. The surface St distributions and the geometry of the separation area were sensitive to the distance between the two jets. The separation area was larger than in the single injection case. Separation area was largest at $S_{Y} / D \sim 8$, while the area of primary heating increased as $S_{Y}$ increased.

\section{Nomenclature}

c: $\quad$ Specific heat capacity, $\mathrm{J} /(\mathrm{kg} \cdot \mathrm{K})$

$c_{p, \text { air }}$ : Specific heat capacity of air, $\mathrm{J} /(\mathrm{kg} \cdot \mathrm{K})$

$D$ : Diameter of injection hole, $\mathrm{mm}$

$k: \quad \alpha / \rho c$, thermal conductivity, $\mathrm{W} /(\mathrm{m} \cdot \mathrm{K})$

$L: \quad$ Length of wind tunnel model, $\mathrm{mm}$

$l_{\text {sep }}$ : Separation length, $\mathrm{mm}$

$M$ : Mach number
$P$ : Pressure, $\mathrm{kPa}$ or $\mathrm{Pa}$

$\dot{q}:$ Heat flux, $\mathrm{W} / \mathrm{cm}^{2}$

$R:$ Nose radius of wind tunnel model, $\mathrm{mm}$

Re: Reynolds number, $\mathrm{m}^{-1}$

$S$ : Spacing between injection holes, $\mathrm{mm}$

St: Stanton number

$T$ : Temperature, $\mathrm{K}$

$t$ : Time, $s$

$U:$ Velocity, $\mathrm{m} / \mathrm{s}$

$X$ : Streamwise coordinate, $\mathrm{mm}$

$\alpha$ : Thermal diffusivity, $\mathrm{m}^{2} / \mathrm{s}$

$\theta$ : Cone half angle of wind tunnel model, degrees

$\rho:$ Density, $\mathrm{kg} / \mathrm{m}^{3}$

$\varphi$ : Angle of rotation, degrees

\section{Subscripts}

0: Total value

$\infty$ : Freestream value

$J$ : Value of injection

$b$ : Value of polyurethane resin

$w$ : Wall value.

\section{Conflicts of Interest}

The authors declare that there are no conflicts of interest regarding the publication of this paper.

\section{Acknowledgments}

The authors would like to thank Professor Hosei Nagano, Mr. Ryohei Fujita, Mr. Jihoon Kim, and Mr. Kimihide 
Odagiri who provided insight and expertise that assisted the measurement of thermal properties of the polyurethane resin. The authors also acknowledge Dr. Hiroshi Ozawa who provided expertise and advice on the temperature sensitive paint technique.

\section{References}

[1] AGARD special course on missiles aerodynamics, AGARD 804, 1994.

[2] T. F. Fric and A. Roshko, "Vortical Structure in the Wake of a Transverse Jet," Journal of Fluid Mechanics, vol. 279, pp. 1-47, 1994.

[3] S. J. Beresh, J. F. Henfling, R. J. Erven, and R. W. Spillers, "Penetration of a transverse supersonic jet into a subsonic compressible crossflow," AIAA Journal, vol. 43, no. 2, pp. 379-389, 2005.

[4] A. Gülhan, G. Schütte, and B. Stahl, "Experimental study on aerothermal heating caused by jet-hypersonic crossflow interaction," Journal of Spacecraft and Rockets, vol. 45, no. 5, pp. 891-899, 2008.

[5] B. Stahl, H. Esch, and A. Gülhan, "Experimental investigation of side jet interaction with a supersonic cross flow," Aerospace Science and Technology, vol. 12, no. 4, pp. 269-275, 2008.

[6] H. E. G. Powrie, G. J. Ball, and R. A. East, "Comparison of the interactions of two and three dimensional transverse jets with a hypersonic free stream," in $A G A R D$, Computational and Experimental Assessment of Jets in Cross Flow 8, (SEE N 9428003 07-34), pp. 94-28003, Computational and Experimental Assessment of Jets in Cross Flow 8 p(SEE N, 1993.

[7] G. M. Buck, A. N. Watkins, P. M. Danehy, J. A. Inman, D. W. Alderfer, and A. A. Dyakonov, "Experimental measurement of RCS jet interaction effects on a capsule entry vehicle," AIAA Paper 1229, 2008.

[8] A. N. Watkins, G. M. Buck, B. D. Leighty, W. E. Lipford, and D. M. Oglesby, "Using pressure- and temperature-sensitive paint for global surface pressure and temperature measurements on the aft-body of a capsule reentry vehicle," AIAA Paper 1230, 2008.

[9] S.-H. Lee, "Characteristics of dual transverse injection in scramjet combustor, part 1: Mixing," Journal of Propulsion and Power, vol. 22, no. 5, pp. 1012-1019, 2006.

[10] A. Kovar and E. Schülein, "Comparison of experimental and numerical investigation on a jet in a supersonic cross-flow," Aeronautical Journal, vol. 110, no. 1108, pp. 353-360, 2006.

[11] A. S. Pudsey, V. Wheatley, and R. R. Boyce, "Behavior of multiple-jet interactions in a hypersonic boundary layer," Journal of Propulsion and Power, vol. 31, no. 1, pp. 144-155, 2015.

[12] M. Kurita, T. Okada, and Y. Nakamura, "Side jet aerodynamic interaction on a blunt body in hypersonic flow," Journal of the Japan Society for Aeronautical and Space Sciences, vol. 50, p. 394, 2003 (Japanese).

[13] M. K. Ibrahim, T. Nakamura, K. Kitamura, K. Mori, and Y. Nakamura, "The Role of vortices in side jet/blunt body interaction at hypersonic speed," Transactions of the Japan Society for Aeronautical and Space Sciences, Space Technology Japan, vol. 7, pp. 1-10, 2009.

[14] T. Liu and J. P. Sullivan, Pressure and Temperature Sensitive Paints, Springer-Verlag, Berlin, Germany, 2005.

[15] H. Nagai, R. Swamura, and K. Asai, "Experimental study of heat transfer measurement using temperature-sensitive paint for high-temperature application in hypersonic flow," AIAA Paper 2011-0850, 2011.

[16] W. J. Cook and E. J. Felderman, "Reduction of data from thinfilm heat-transfer gages - a concise numerical technique," AIAA Journal, vol. 4, no. 3, pp. 561-562, 1966.

[17] R. Abgrall, J.-A. Désidéri, R. Glowinski, M. Mallet, and J. Périaux, Hypersonic Flows for Reentry Problems, vol. 3, Springer Berlin Heidelberg, Berlin, Heidelberg, 1992.

[18] L. Lees, "Laminar heat transfer over blunt-nosed bodies at hypersonic flight speeds," Journal of Jet Propulsion, vol. 26, no. 4, pp. 259-269, 1956.

[19] J. A. Fay and R. Riddell, "Theory of stagnation point heat transfer in dissociated air," Journal of the Aeronautical Sciences, vol. 25, pp. 73-85, 1958.

[20] R. Kawamura, T. Sawada, and K. Seki, "Study of lattice of cireular cylinders in supersonic and hypersonic flow I. Drag of lattice of two parallel circular cylinders in supersonic fiow," Journal of Aeronautical Research Institute, The University of Tokyo, vol. 3, no. 6A, pp. 283-318, 1963 (Japanese).

[21] R. Kawamura, T. Sawada, and K. Seki, "Study of lattice of cireular cylinders in supersonic and hypersonic flow 2. Shock waves about lattices of two parallel circular cylinders in supersonic flow," Journal of Aeronautical Research Institute, The University of Tokyo, vol. 3, no. 7, pp. 619-630, 1963 (Japanese).

[22] A. Chpoun and D. Passerel, "Reconsideration of oblique shock wave reflections in steady flows. part 1. experimental investigation," Journal of Fluid Mechanics, vol. 301, pp. 19-35, 1995. 


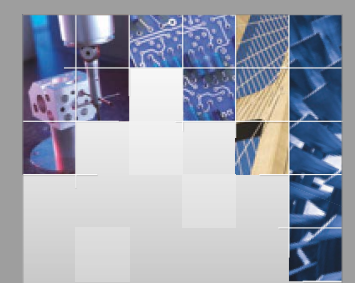

\section{Enfincering}
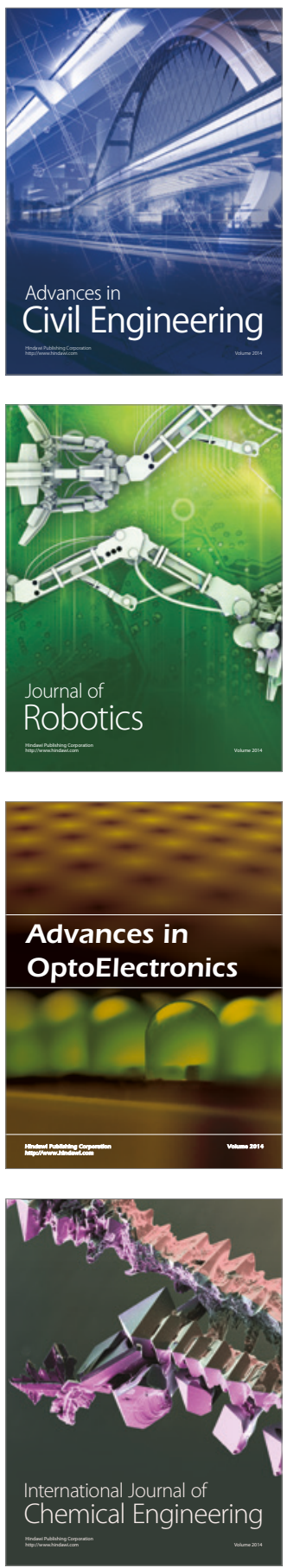

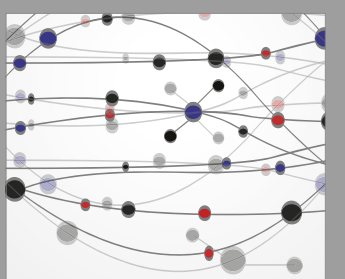

The Scientific World Journal

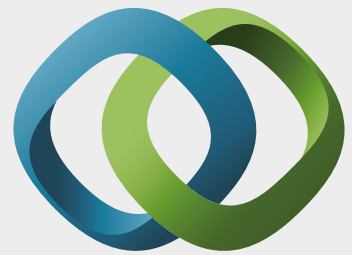

\section{Hindawi}

Submit your manuscripts at

https://www.hindawi.com
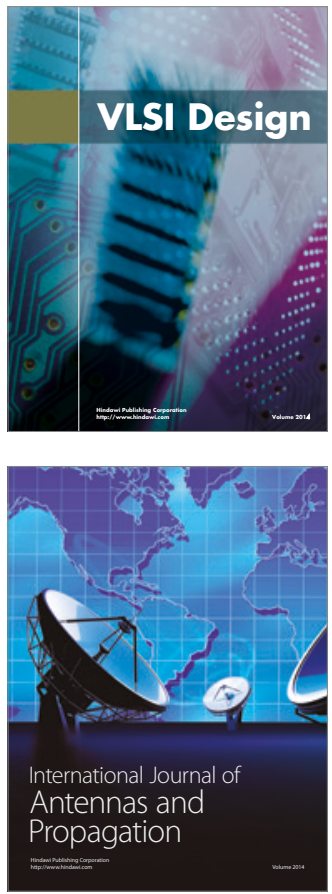

\section{Rotating}

Machinery
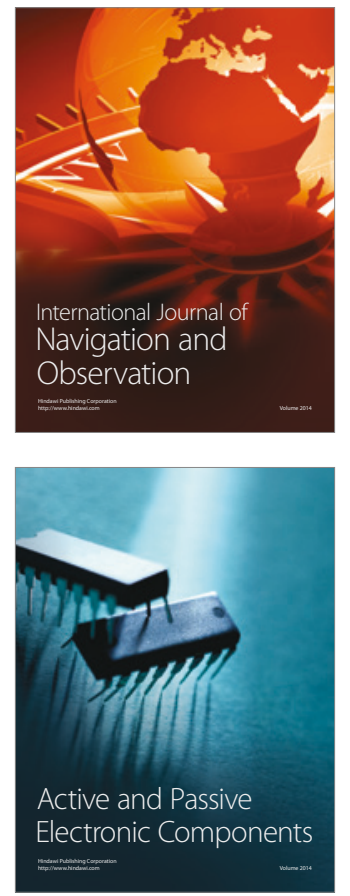
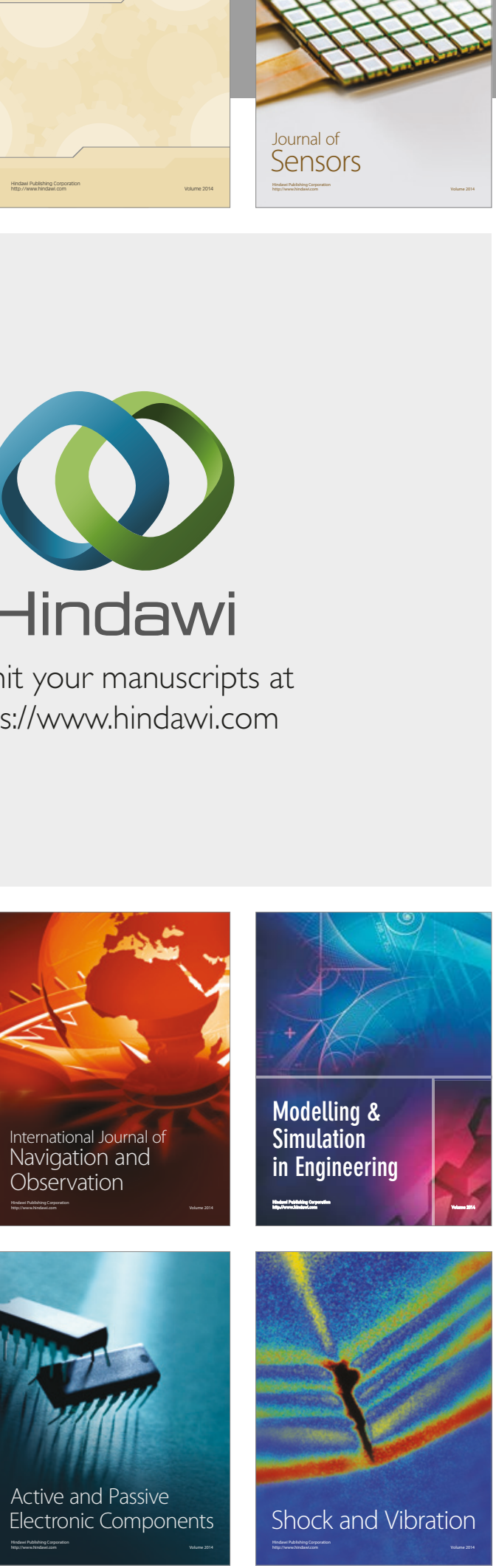
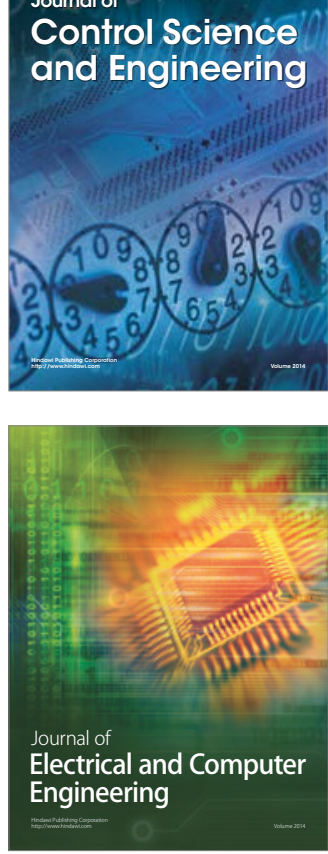

Distributed

Journal of

Control Science

and Engineering
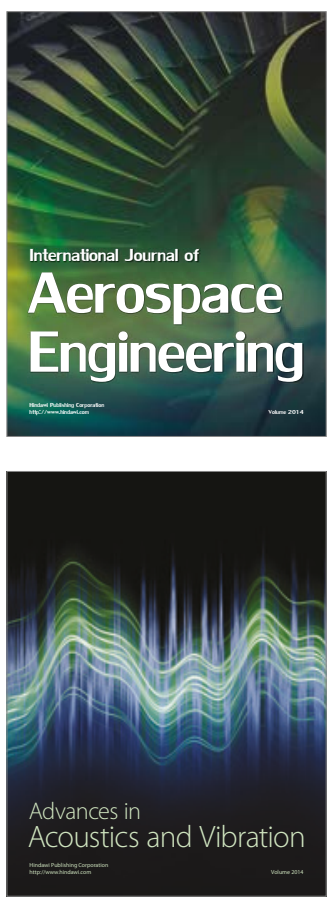

Sensor Networks 